intermittent drug regimens for advanced breast cancer. ${ }^{1}{ }^{3}$ Furthermore, once the disease becomes unresponsive to multiple drug combinations it is difficult to give further effective chemotherapy to patients who have been exposed to major cytotoxic agents. The development of independent non-cross-resistant combinations is therefore of practical importance.

Our two different combinations proved equally effective in terms of response rate, duration of remission, and median survival. On both treatments the median survival of complete plus partial responders was significantly longer than that of patients with no response or only objective improvement. Considering both duration of response and median survival our results are comparable to those reported with similar or different combinations.

Our findings indicate that in patients with advanced breast cancer previously untreated with chemotherapy a two-drug combination (AV) is as effective as a three-drug regimen (CMF). The results of this and other prospective studies ${ }^{6}{ }^{7}$ raise the question of how many drugs and which ones are needed to induce a satisfactory remission rate. The observation that complete plus partial remissions obtained with AV were maintained and even improved by CMF indicates that the duration of response was prolonged by a non-cross-resistant combination when primary treatment included adriamycin. It is well known that treatment with adriamycin cannot be safely continued once the total dose exceeds $500-600 \mathrm{mg} / \mathrm{m}^{2}$. Although secondary treatment is usually less effective than primary treatment, we can conclude that no cross resistance exists between CMF and AV.

Both treatments produced partial and even complete radiographic healing of osteolytic bone metastases in about a quarter of patients. This result, which has previously been noted only after endocrine treatment, can now be obtained also by prolonged combination regimens. Owing to the limited number of patients the question of the influence of free interval and menopausal status on the response rate cannot be answered fully. Available data suggest, however, that these pretreatment variables did not significantly influence the incidence of complete plus partial response.
Considering our results within the framework of present combined drug regimens for advanced breast cancer, we conclude that although combination chemotherapy has improved the control of disease compared with single-agent treatment, no multiple drug regimen has so far proved to be definitely better than another in terms of duration of response and survival. From this point of view, combination chemotherapy alone seems to have almost reached a plateau in its capacity to control disseminated breast cancer. While the initial promising results of treatment with synergistic combinations ${ }^{4}{ }^{5}$ should be confirmed by long-term analysis, new possibilities to improve the duration of response may lie in appropriate sequential combinations or in combining multiple-drug regimens with immunotherapy ${ }^{9}$ or endocrine therapy. The extensive tumour burden present in most patients with clinically disseminated breast cancer presents an unfavourable kinetic condition to available chemotherapeutic combinations. Nevertheless, the therapeutic approaches mentioned above are more likely to improve the duration of remission and survival of responders than to increase the response rate.

This work was supported in part by contract NO 1-CM-33714 with the National Cancer Institute. National Institutes of Health, Division of Cancer Treatment, USA.

Requests for reprints should be addressed to Dr G Bonadonna, Istituto Nazionale Tumori, Via Venezian 1, Milan 20133, Italy.

\section{References}

${ }^{1}$ Broder, L E, and Tormey, D C, Cancer Treatment Reviews, 1974, 1, 183. 2 De Lena, M, et al, Cancer, 1975, 35, 1108.

${ }^{3}$ Canellos, G P, et al, Annals of Internal Medicine, in press.

4 Jones, S E, Durie, B G M, and Salmon, S E, Cancer, 1975, 36, 90.

${ }_{5}$ Blumenschein, G R, et al, Proceedings of the American Association for Cancer Research, 1974, 15, 193.

${ }^{6}$ Ahman, D L, et al, Cancer Chemotherapy Reports, 1974, 58, 877.

7 Gottlieb, J A, et al, Adriamycin Review, EORTC International Symposium. European Press Medikon, 1975.

${ }^{8}$ Mantel, M, Biometrics, 1967, 23, 65.

9 Cardenas, J O, et al, Cancer Immunology and Immunotherapy, 1976, in press.

\title{
Rheumatoid arthritis and ankylosing spondylitis occurring together
}

\author{
GEORGES H FALLET, MICHAEL MASON, HEDLEY BERRY, ALASTAIR G MOWAT, \\ IRADJ BOUSSINA, JEAN-CHARLES GERSTER
}

British Medical fournal, 1976, 1, 804-807

\section{Summary}

Rheumatoid arthritis and ankylosing spondylitis are often difficult to differentiate, though it is important to

\section{Division de Rhumatologie, Hôpital Beau-Séjour, Geneva}

G H FALLET, MD, professor

I BOUSSINA, $\mathrm{MD}, 1$ st senior registrar

J-C GERSTER, MD, 2nd senior registrar

Department of Rheumatology, The London Hospital, London E1 1BB

MICHAEL MASON, DM, FRCP, consultant physician

H BERRY, DM, MRCP, senior registrar (now consultant physician, Department of Rheumatology, King's College Hospital, London SE5)

Department of Rheumatology, Nuffield Orthopaedic Centre, Headington, Oxford

A G MOWAT, MB, FRCP, consultant rheumatologist do so as the natural history and treatment of the two conditions differ. Nine patients have recently been seen, each of whom fulfilled the criteria for both rheumatoid arthritis and ankylosing spondylitis. In eight of the nine patients the histocompatibility antigen HLA-27 was present. A possible explanation of these cases is that one of the diseases occurred by chance in patients already suffering from the other, but this is extremely unlikely. If a chance association is not the correct explanation the basic concepts defining rheumatoid arthritis and ankylosing spondylitis must be reconsidered.

\section{Introduction}

The distinction between rheumatoid arthritis and ankylosing spondylitis has relied considerably on the results of the RoseWaaler and latex tests, which detect the IgM component of rheumatoid factor. Despite their well-recognised defects of false-positives and occasional false-negatives, these remain 
universally used routine tests. In Europe rheumatoid arthritis and ankylosing spondylitis are accepted as being distinct and separate diseases on the basis of clinical, radiological, and pathological differences, and the absence of rheumatoid factor in the serum of patients with ankylosing spondylitis has supported this view. Confidence that ankylosing spondylitis is a separate disease is reflected, for example, in the statement by de Seze and Ryckawaert ${ }^{1}$ that all that is needed for its diagnosis is a finding of bilateral sacroiliitis and a raised sedimentation rate. None the less, some practical difficulties do exist as up to half the patients with ankylosing spondylitis have peripheral joint involvement during their disease and the spine may be more or less spared. ${ }^{2}$ It has been claimed also that about $30 \%$ of patients with rheumatoid arthritis may have sacroiliitis, often bilateral. ${ }^{3}+$ In clinical practice, therefore, diagnosis is an important problem since the natural history and complications of the two diseases differ and there are important differences in treatment.

Against this background the recognition of the significance of the histocompatibility antigen distribution provided a new landmark. Thus the independent demonstrations by Brewerton et $a l^{5}$ and Schlosstein $e t a l^{6}$ of an association between HLA-27 and ankylosing spondylitis in $90 \%$ of cases, this antigen being found in only $6^{\circ}{ }_{0}$ of the normal population and $9^{\circ}{ }_{0}$ of a typical rheumatoid population, was an important observation. ${ }^{7}$

In clinical practice diagnostic problems usually arise in two types of patient: those with a peripheral polyarthritis who also have bilateral sacroiliitis and a positive latex test result, and those with spondylitis and also an extensive peripheral polyarthritis. We report here on nine patients who fell into one or other of these categories and suggest that finding the HLA-27 antigen may help to clarify the situation.

\section{Case reports}

Case 1-This 64-year-old man initially presented with low backache in 1941, when he was 30. In 1946 he developed iritis. In 1950 he had atlantoaxial subluxation requiring surgical correction. He was then noted to have temporomandibular joint and knee arthritis on both sides. In 1969 he developed peripheral polyarthritis affecting wrists, proximal interphalangeal joints, and shoulders. In 1972 both hips also became affected. On examination he was found to have extensive polyarthritis affecting proximal interphalangeal joints, metacarpophalangeal joints, wrists, metatarsophalangeal joints, and knees. There were no nodules and there was no spinal movement. $X$-ray examinations showed extensive peripheral erosive arthropathy. Both sacroiliac joints were ankylosed, and there was squaring of vertebral bodies with syndesmophyte formation and early bridging. Erythrocyte sedimentation rate (ESR) was $72 \mathrm{~mm}$ in one hour and the latex test titre was 1/640. Antigen HLA-27 was present.

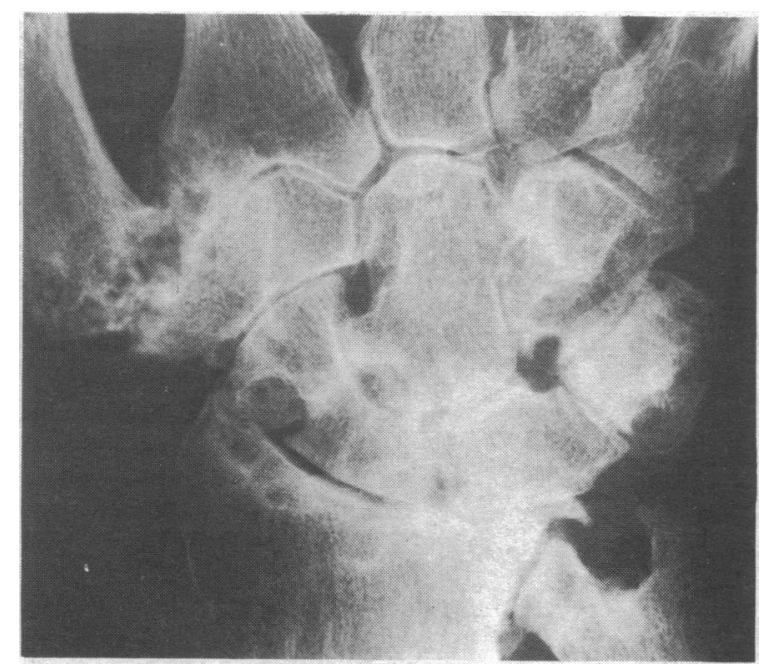

FIG 1-Case 2. Radiograph of right wrist showing sever erosive arthritis.

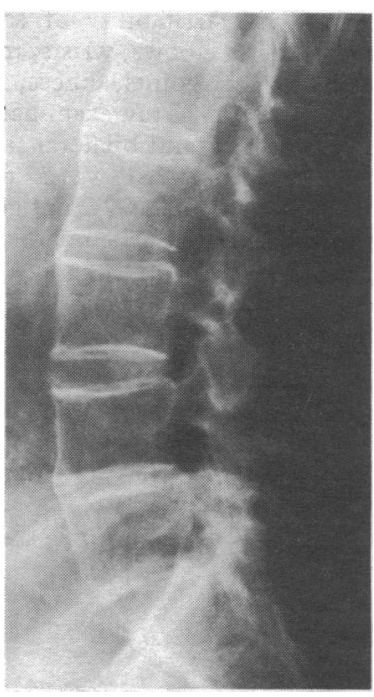

FIG 2-Case 3. Lateral radiograph of lumbar spine showing complete bridging.

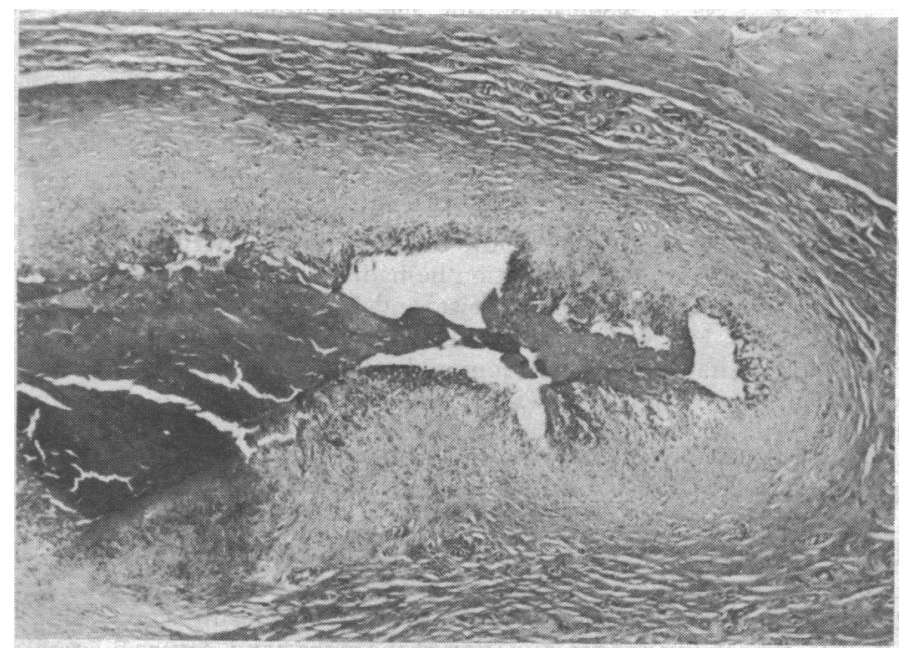

FIG 3-Case 3. Histological appearances of rheumatoid nodule on left elbow $(\mathrm{H}$ and $\mathrm{E} \times 7 \cdot 6$.

Case 2-This 68-year-old man had a brief isolated episode of diarrhoea in 1936, when he was 29 , followed by transient arthritis of knees and left foot. In 1943 he developed further transient arthritis of the knees. In 1946 he had an attack of iritis and was noted to have limited spinal movement. In 1967 and 1968 painful limitation of shoulders and hips developed, but there was no sacroiliac pain. There was also no urethritis. Examination showed a rigid kyphotic spine and widespread peripheral arthritis. Radiographs showed ankylosis of the sacroiliac joints and extensive erosive changes in hands, wrists (fig 1), and shoulders. ESR was $107 \mathrm{~mm}$ in one hour and the latex test titre was 1/640. Antigen HLA-27 was present.

Case 3-In 1973 this 68-year-old woman developed a swelling of the middle left metacarpophalangeal joint, which was followed by involvement of the remaining metacarpophalangeal joints of both hands with early morning stiffness. Over the next two months the swelling spread to affect elbows, knees, shoulders, and left wrist. She had no backache. In February 1974 a nodule appeared on the left elbow. On examination her spine was completely immobile. Both knees, the metacarpophalangeal joints, and index, ring, and middle fingers of both hands were swollen. Radiographs showed severe bilateral sacroiliitis, and spinal ankylosis (fig 2). The joints of the hands showed loss of space but no erosions. ESR was $55 \mathrm{~mm}$ in one hour, the latex test titre was $1 / 640$, and HLA-27 antigen was present. A biopsy of the nodule showed classical rheumatoid change (fig 3 ).

Case 4-This man, born in 1922, developed inflammatory changes of the metatarsophalangeal joints and knees when he was 40 . This was later followed by changes in the proximal interphalangeal joints, 
wrists, elbows, and shoulders. Morning stiffness was a pronounced feature and nodules developed on both elbows. Examination showed limited spinal movement with irritability of sacroiliac joints and polyarthritis affecting shoulders, elbows, wrists, metacarpophalangeal joints, proximal interphalangeal joints, knees, ankles, metatarsophalangeal joints, and the left hip. $X$-ray examination showed erosive arthropathy of the hands and feet and bilateral sacroiliitis with bony sclerosis bridging at the level of L 4. ESR was $57 \mathrm{~mm}$ in one hour, the latex test titre was 1/2560, and HLA-27 antigen was present. A biopsy of the nodule showed classical rheumatoid change (fig 4).

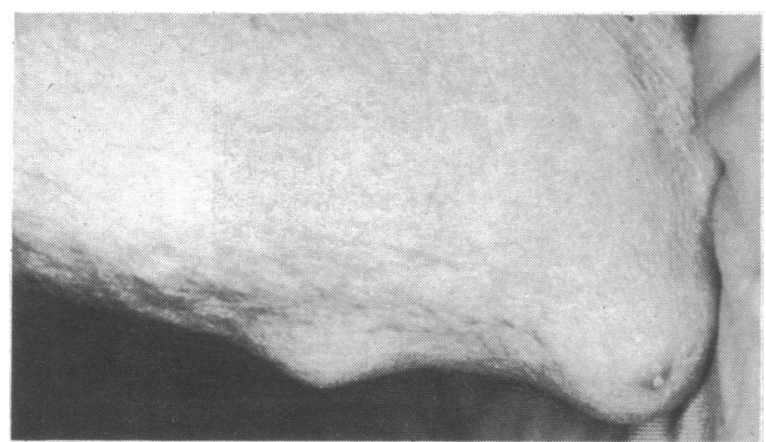

FIG 4-Case 4. Nodular bursitis and rheumatoid nodule on left elbow.

Case 5-This 67-year-old man developed polyarthritis of the hands, wrists, shoulders, knees, and ankles with Raynaud's phenomenon when he was 57 . In 1974 hips, knees, and shoulders became affected and he lost $9 \mathrm{~kg}$. He suffered low backache and underwent hip replacement in 1975. On examination he had inflammatory arthropathy affecting shoulders, hips, right wrist, and hand with ulnar deviation of both hands. He also had limited cervical and thoracic spinal movement and irritable sacroiliac joints. Radiographs showed bilateral sacroiliitis with bony sclerosis, syndesmophyte at $T 12$, vertebral bridging at $\mathrm{L} 2 / \mathrm{L} 3$ (fig 5), and severe erosive changes in hands and wrists. ESR was $93 \mathrm{~mm}$ in one hour, latex test titre was $1 / 80$, and HLA-27 antigen was absent.

Case 6-This 75-year-old woman developed polyarthritis of hands, wrists, shoulders, knees, ankles, and neck with early morning stiffness in 1963, when she was 63. She gave a history of pain in the neck and low back over many years. Her son had ankylosing spondylitis. Examination showed ulnar deviation of the right hand, severe bilateral wrist arthropathy, effusion of right knee, no nodules, and limitation

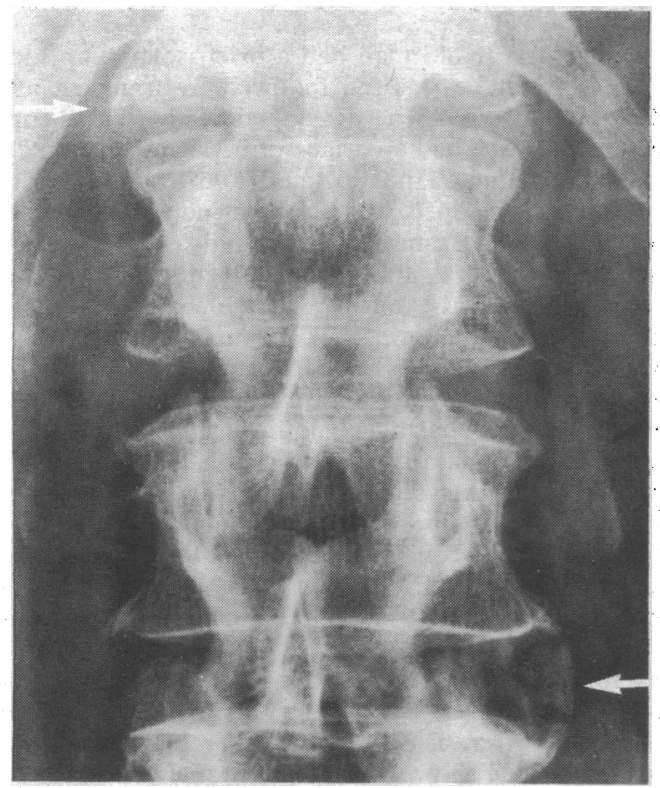

FIG 5-Case 5. Radiograph of thoracolumbar junetion showing syndesmophyte at $\mathrm{T} 12$ and vertebral bridging at $\mathrm{L} 2-\mathrm{L} 3$. of spinal movement. Radiographs showed erosive changes in hands but not feet, ankylosing changes in the left wrist, obliteration of sacroiliac joints, and syndesmophyte formation and bridging of the lower dorsal spine. ESR was $50 \mathrm{~mm}$ in one hour, latex test titre was $1 / 160$, and HLA-27 antigen was present.

Case 7-This woman, born in 1918, developed iritis in 1960, symmetrical polyarthritis of hands in 1969 , involvement of the left knee and later the right knee in 1973, and became incapacitated in 1974, needing a stick. She did not have backache. Examination showed grossly limited movement of the lumbar spine and a flexion deformity of the right elbow. There was bilateral synovial thickening of the wrists and involvement of the metacarpophalangeal joints with synovial thickening. In both knees there was a fixed flexion deformity of $25^{\circ} . X$-ray examination showed extensive erosive changes of the hands and feet, bilateral sacroiliitis, and cervical spondylosis. ESR was $71 \mathrm{~mm}$ in one hour, the latex test titre was $1 / 320$, and HLA-27 antigen was present. A synovial biopsy of left knee showed changes consistent with rheumatoid arthritis.

Case 8-This 62-year-old man developed sacroiliac pain when he was 29 . By the time he was 35 this had spread to affect all the spine and he was given deep $x$-ray therapy. In 1950 , when he was 37 , his temporomandibular joint became painful. In 1953 he developed symmetrical polyarthritis of peripheral joints with early morning stiffness. On examination he had grossly limited spinal movement. There was ulnar deviation at metacarpophalangeal joints with subluxation and extensive muscle wasting. Radiographs showed complete sacroiliac fusion with spinal ankylosis extending to the cervical region (fig 6). There were also extensive erosive changes in hands and feet. ESR was $14 \mathrm{~mm}$ in one hour, the latex test titre was $1 / 320$, and HLA-27 antigen was present.

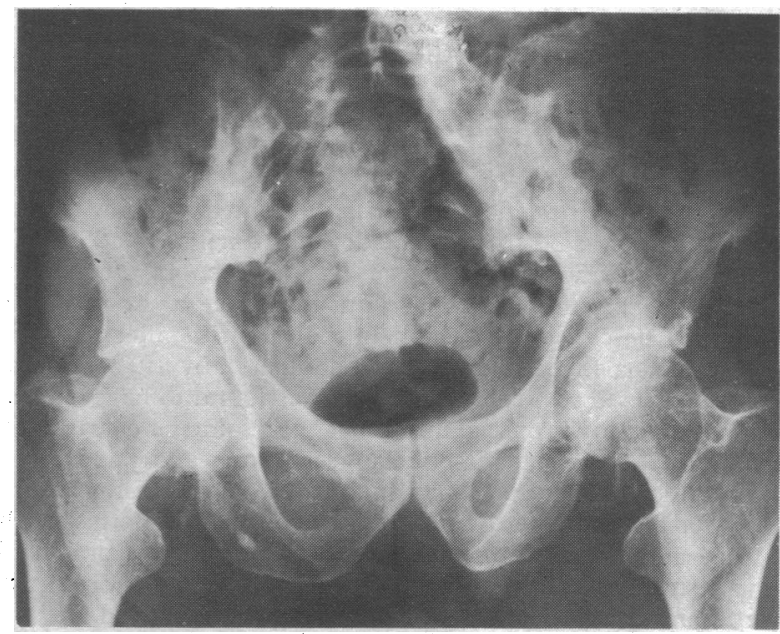

FIG 6-Case 8. Radiograph of sacroiliac joints showing complete fusion.

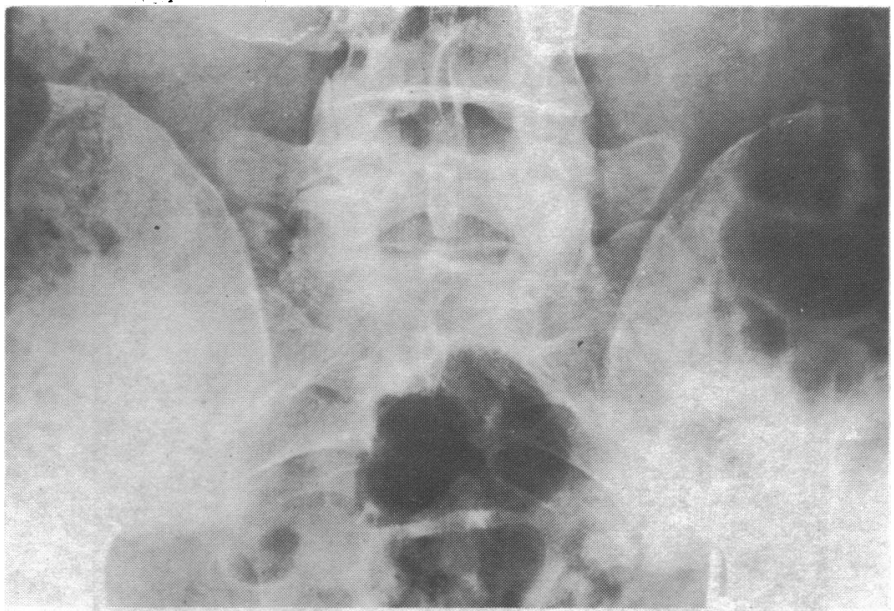

FIG 7-Case 9. Radiograph of sacroiliac joint showing moderate bilateral sacroiliitis. 
Case 9-In 1968 this 44-year-old man complained of pain in both thighs; this was relieved by phenylbutazone. During the next few years he developed recurrent low back pain with morning stiffness that was relieved by indomethacin. In 1972 peripheral polyarthritis began in the index metacarpophalangeal joints and became widespread, affecting hands, feet, elbows, knees, and shoulders. In 1975 he suffered increasing backache. Examination showed limited lumbar and cervical spinal movement. He had active symmetrical peripheral polyarthritis with synovitis, and he developed two rheumatoid nodules, one on the extensor aspect of the forearm and another on a finger. Radiographs showed considerable bilateral sacroiliitis (fig 7) and mild erosive changes in hands and feet. ESR was $62 \mathrm{~mm}$ in one hour, the latex test gave a positive result (differential agglutination titre was 1/2048), he was negative for antinuclear factor, and HLA-27 antigen was present.

\section{Discussion}

The classification of these nine patients is difficult. They do not fall into the group of "seronegative spondarthritides" described by Moll et al, ${ }^{8}$ for they all had seropositive disease. None had a history of urethritis, psoriasis, or colitis. Eight of the nine patients had the histocompatibility antigen HLA-27 on their lymphocytes, the expected proportion in patients with ankylosing spondylitis. ${ }^{5}{ }^{9}$ About $9 \%$ of a rheumatoid population would be expected to exhibit HLA-27 histocompatibility antigen, ${ }^{7}$ but this does not mean that these patients will all have sacroiliitis any more than such a finding would in the ordinary population.

The picture presented by the appearance of spinal ankylosis (and widespread peripheral joint ankylosis) in elderly rheumatoid patients leading a sheltered life in long-stay hospitals is also different. Only one of 11 such patients reported by Calin et $a l^{10}$ and Grahame $e t a l^{11}$ had the histocompatibility antigen HLA-27. Conlon et al ${ }^{12}$ have shown that it is rare for ankylosis to occur in rheumatoid arthritics.

Our nine cases all fulfilled the diagnostic criteria of the American Rheumatism Association for both rheumatoid arthritis ${ }^{13}$ and ankylosing spondylitis. ${ }^{2}$ One explanation of this phenomenon is that rheumatoid arthritis had occurred by chance in patients already suffering from ankylosing spondylitis-for example, cases $1,2,6,7$, and 9-or, conversely, that ankylosing spondylitis had developed by chance in patients already suffering from rheumatoid arthritis-for example, case 5 . In cases 3 and 4 the spondylitis was asymptomatic and the time of onset could not be identified. Severe erosive rheumatoid arthritis occurs in about 1 in 100 of the general population, ${ }^{14-16}$ and ankylosing spondylitis occurs in 1 in 500 to 1 in $2000,{ }^{17} 18$ so, assuming the two diseases are independent of each other, the chance of this association in the same person is about 1 in 50000 to 1 in 200000 and, by the same token, in nine cases, about 1 in 450000 to 1 in 1800000 . Hence if our cases were not the result of a simple coincidence between rheumatoid arthritis and ankylosing spondylitis one would be forced to reconsider the basic concepts that, at present, define these two diseases.

We thank Professor R Lagier, department of pathology, Dr M Jeannet, charge de cours, immunology of transplantation unit, and Dr E Meyer, consultant in rheumatology (all of whom are from Geneva), for their valuable contribution to this work.

\section{References}

${ }^{1}$ Seże, S de, and Ryckewaert, A, Maladies des Os et des Articulations, p 731. Paris, Flammarion, 1965.

2 Wright, V, and Moll, J M H, British fournal of Hospital Medicine, 1973, 9, 331 .

${ }^{3}$ Fallet, G H, et al, Revue du Rhumatismect des Maladies Ostéo-articulaires, $1970,37,213$.

4 Ott, V R, Schmidy, K, and Herberholz, G, in Congresso Europeu de Reumatologia, p 168. Lisboa, Instituto Portagues de Reumatologia, 1967. 5 Brewerton, D A, et al, Lancet, 1973, 1, 904.

6 Schlosstein, L, et al, New England fournal of Medicine, 1973, 288, 704.

7 Kueppers, F, Brackertz, D, and Mueller-Eckhardt, C, Lancet, 1972, 2, 1425.

${ }^{8}$ Moll, J M H, et al, Medicine, 1974, 53, 343.

${ }^{9}$ Morris, R I, et al, New England fournal of Medicine, 1974, 290, 111.

10 Calin, A, et al, Lancet, 1974, 1, 874.

11 Grahame, R, et al, Rheumatology and Rehabilitation, 1975, 14, 25.

12 Conlon, P W, Isdale, I C, and Rose, B S, Annals of the Rheumatic Diseases, 1966, 25, 120.

${ }^{13}$ Ropes, M W, et al, Bulletin on Rheumatic Diseases, 1958, 9, 175.

14 Lawrence, J S, et al, Annals of the Rheumatic Diseases, 1966, 25, 425.

15 Mikkelsen, W M, et al, fournal of Chronic Diseases, 1967, 20, 351.

16 Wagenhäuser, F J, Die Rheumamorbidität. Bern, Huber, 1969.

17 Blécourt, J J de, Polman, A, and Blécourt-Meindersma, I de, Annals of the Rheumatic Diseases, 1961, 20, 215.

18 West, H F, Annals of the Rheumatic Diseases, 1949, 8, 143.

\title{
False-positive lung scans and radiotherapy
}

\author{
N T BATEMAN, D N CROFT
}

British Medical fournal, 1976, 1, 807-808

\begin{abstract}
Summary
It has been suggested that obliterative vasculitis in the lung might mimic pulmonary embolism on a ventilationperfusion scan. The combined scans of six patients with breast cancer who had undergone radiotherapy to the chest wall, which can induce pulmonary vasculitis, were therefore examined. Eleven of the 12 scans showed
\end{abstract}

\footnotetext{
Department of Nuclear Medicine, St Thomas's Hospital, London SE1 7EH

N T BATEMAN, MRCP, medical registrar

D N CROFT, DM, FRCP, consultant physician
}

perfusion defects with ventilation-perfusion mismatch on the irradiated side. Special care is needed in interpreting the lung scans of patients who have received an appreciable tissue dose of radiation to the lungs; mismatch need not indicate pulmonary embolism.

\section{Introduction}

The combined ventilation-perfusion lung scan is more accurate than the perfusion scan alone in diagnosing pulmonary embolism. $^{1-3}$ It has been suggested ${ }^{3}$ that diseases producing an obliterative vasculitis in the lung might show ventilationperfusion mismatch on the combined lung scan and might be confused with pulmonary embolism. We therefore studied the combined lung scans of patients who had undergone radiotherapy that included a significant tissue dose to the lung, which is known to induce pulmonary vasculitis. ${ }^{4}$ 